\title{
Anelasticity of Phase Transitions and Magnetostriction in Fe-(27-28\%)Ga Alloys
}

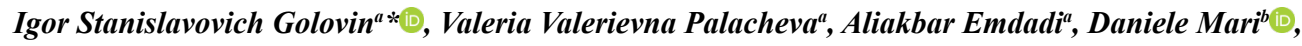

\author{
Alexei Heintz, Anatoliy Mikhailovich Balagurov ${ }^{c}$, Ivan Anatolievich Bobrikov \\ ${ }^{a}$ National University of Science and Technology MISiS, Moscow, Russia \\ ${ }^{b}$ Ecole Polytechnique Fédérale de Lausanne, Lausanne, Suisse \\ 'Joint Institute for Nuclear Research, Dubna, Russia
}

Received: October 05, 2017; Revised: February 21, 2018; Accepted: April 20, 2018

Several aspects of anelastic behavior of Fe-(27-28\%)Ga-Tb alloys are considered in this paper: (i) the phase transitions from a metastable to an equilibrium phase and phase transitions between equilibrium phases at higher temperatures in as cast alloys, (ii) the nature of corresponding three transient anelastic effects, (iii) the formation of an intrinsic composite microstructure with a different ratio between the bcc-derivative metastable and fcc-derivative equilibrium phases that have different magnetostriction and the effect of alloys doping by $\mathrm{Tb}$ to stabilize the metastable phase with high positive values of magnetostriction.

Keywords: Fe-Ga, mechanical spectroscopy, transient effects, magnetostriction, neutron diffraction, phase transitions.

\section{Introduction}

Fe-Ga alloys exhibit attractive functional properties, such as magnetostriction that can be varied from giant positive values to negative values including zero magnetostriction ${ }^{1,2}$. This effect relates to a complex phase transformation sequences in the $\mathrm{Fe}-27 \% \mathrm{Ga}$ alloys. Phase transitions in $\mathrm{Fe}_{3} \mathrm{Ga}$-type alloys were first studied by X-ray diffraction ${ }^{3-5}$, and more recently by in situ neutron diffraction ${ }^{2,6,7}$ to characterize the bulk alloy. Different ratios between metastable and equilibrium phases characterized by magnetostriction values of different signs vary the magnetostriction values of the alloy, $\lambda_{\mathrm{s}}$, from positive to negative, including the value of $\lambda_{\mathrm{s}}=0^{2}$. The phase transition from metastable $\mathrm{D}_{3}$ to stable $\mathrm{L}_{2}$ is accompanied with a well-pronounced transient anelastic effect at about $450-550^{\circ} \mathrm{C}$ depending on the heating rate ${ }^{8}$. Anelastic effects for phase transitions at higher temperatures have as yet not been reported in the literature. Doping Fe-Ga with rare earth elements enhances magnetostriction in Fe-(17-19)Ga alloys by creating local inhomogeneities ${ }^{9-11}$ and stabilizes the metastable bcc-derivative phase in as cast Fe-27Ga alloys by preventing $\mathrm{L}_{2}$ phase nucleation on the $\mathrm{D} 0_{3}$ grain boundaries $^{7,12-14}$. The structure of metastable Fe-27Ga type alloys before their transition to equilibrium $\mathrm{L}_{2}$ phase may show several metastable phases $\left(\mathrm{A} 2, \mathrm{~B} 2, \mathrm{D} 0_{3}\right)$, the size of which, as reported in the literature, can be from nano (e.g. ${ }^{15}$ ) to macro domains.
In this paper, we studied the following phenomena: (i) transient internal friction peaks accompanied by three firstorder phase transitions, namely: $\mathrm{D}_{3}$ to $\mathrm{L}_{2}, \mathrm{~L}_{2}$ to $\mathrm{D} 0_{19}$, and $\mathrm{D} 0_{19}$ to $\mathrm{A} 2$, (ii) influence of doping by $\mathrm{Tb}$ on these transitions, (ii) tailoring magnetostriction by isothermal phase transitions in $\mathrm{Fe}-27 \% \mathrm{Ga}$ alloys.

\section{Methods}

Several Fe-27at.\%Ga and Fe-27.4at.\%Ga-Tb alloys were produced by rapid solidification in a copper mold using pure Fe and Ga by arc melting under the protection of a high-purity inert argon gas using an Arc 200 mini vacuum furnace (Arcast Inc, USA). In this paper, all the compositions are given in atomic percent. We used energy dispersive spectroscopy to measure the chemical compositions of the cast buttons with $\pm 0.2 \%$ accuracy as 27.4 to $27.8 \% \mathrm{Ga}$ in binary alloys and Fe-27.4\%Ga-0.3\% Tb.

The phase transitions were characterized by in situ neutron diffraction measured with a high-resolution Fourier diffractometer (HRFD) ${ }^{16}$ at the IBR-2 pulsed reactor in JINR (Dubna). The tests were performed on rectangular samples with a size of $4 \times 8 \times 40 \mathrm{~mm}$. The acquisition time for each diffraction pattern was $1 \mathrm{~min}$. Heating of the samples was carried out in a specialized furnace (ILL standard) with vanadium screens up to $850^{\circ} \mathrm{C}$ at a temperature rate of 1 or $2 \mathrm{~K} / \mathrm{min}$. Further details can be found in Refs. ${ }^{2,6}$. 
The internal friction (IF, or $Q^{-1}$ ) was obtained by measuring the phase lag $\varphi$ between the applied cyclic stress and the resulting strain: $\sigma=\sigma_{0} \cos (\omega t)$ and $\varepsilon=\varepsilon_{0} \cos (\omega t+\varphi)$, correspondingly. $\omega=2 \pi f$ and $\varphi$ is the phase or the loss angle. It can be demonstrated that $\mathrm{IF}=\tan (\varphi)$ if $\varphi$ is small. The measurements were performed by two types of equipment:

1. An inversed torsion pendulum working in a forced mode. Most of the spectra were recorded at a frequency of $0.02 \mathrm{~Hz}$ to enhance the peak amplitude in case of phase transformations. The strain amplitude of the oscillations was $5 \times 10^{-5}$. Additionally, the spectra were also recorded at 0.3 and $1 \mathrm{~Hz}$. The sample size was $1 \times 4 \times 30 \mathrm{~mm}$ between the clamps. The temperature was recorded by a thermocouple inserted in the lower clamp and touching the specimen. Therefore, a relatively good accuracy $( \pm 2 \mathrm{~K})$ can be expected. The heating and cooling rate was $1 \mathrm{~K} / \mathrm{min}$. All the measurements were performed under a vacuum better than $5 \times 10^{-5}$ mbar. Each cycle presented here was confirmed at least once by another measurement in the same pendulum and sometimes even in another apparatus.

2. A dynamical mechanical analyzer DMA Q800 TA Instruments. The measurements were conducted as a function of temperature between 0 and $600{ }^{\circ} \mathrm{C}$ using forced bending single cantilever vibrations in a range between 0.1 and $30 \mathrm{~Hz}$ with $\varepsilon_{0}=7 \times 10^{-5}$ with a heating and cooling rate of 1 or $2 \mathrm{~K} / \mathrm{min}$.

A DualScope C26 magnetic force microscope, MFM (DME Company, Copenhagen, Denmark) was used to obtain the magnetic force gradient image with a lift height of 250 $\mathrm{nm}$ and a high-moment Co-coated tip magnetized normal to the sample surface. The magnetostriction was measured using a hand-made experimental setup based on a strain gauge method up to a saturated magnetic field value of $350 \mathrm{kA} / \mathrm{m}$.

\section{Results}

\subsection{Anelastic effects due to phase transitions}

Several internal friction (IF) peaks of different nature can be observed at Temperature Dependent IF (TDIF) curves for the Fe-27Ga-type alloys. At least three of them are Debye-type thermally activated peaks (P1, P2, P3, P4) and three transient effects $(\operatorname{Tr} 1, \operatorname{Tr} 2, \operatorname{Tr} 3)$ (Fig. 1).

The activation parameters for $\mathrm{P} 1$ and $\mathrm{P} 2$ peaks were collected in our previous papers, e.g. ${ }^{8,17}$, and assigned to Snoek-type and Zener relaxations.

In this paper, we focus on transient effects: in situ neutron diffractions were run with practically the same heating rate as mechanical spectroscopy to define phase transitions in the alloys (Fig. 2). Figure 2 shows intervals for phase transitions, phase transition reaction rates, and atomic volume of the phases in $\mathrm{Fe}-27 \mathrm{Ga}$. The $\mathrm{DO}_{3} \rightarrow \mathrm{L}_{2}$ transition is accompanied by a pronounced jump in the atomic volume, the $\mathrm{D} 0_{19} \rightarrow$ A2 transition is also accompanied with a jump in the atomic volume, whereas this effect is negligibly small in the case of the $\mathrm{L}_{2} \rightarrow \mathrm{D} 0_{19}$ transition. The change in the atomic volume in the range of the co-existing phases ( $\mathrm{DO}_{3}$ and $\mathrm{L}_{2}$ or $\mathrm{D} 0_{19}$ and A2) leads to an increase in micro stresses in the alloy.

It is important to note that each time the transient IF peak is associated with a modulus dip. The drop in the elastic shear modulus is due to a strong coupling between the stress and the anelastic strain: similarly to martensitic transformation, phase interfaces in Fe-Ga alloys propagate during the transformation. Misfit dislocations migration may also contribute. From the temperature of the modulus dip and considering the precision of the measurements, we cannot state that some precursor soft mode activates the transformation. Below we consider anelastic effects accompanied by these phase transitions.

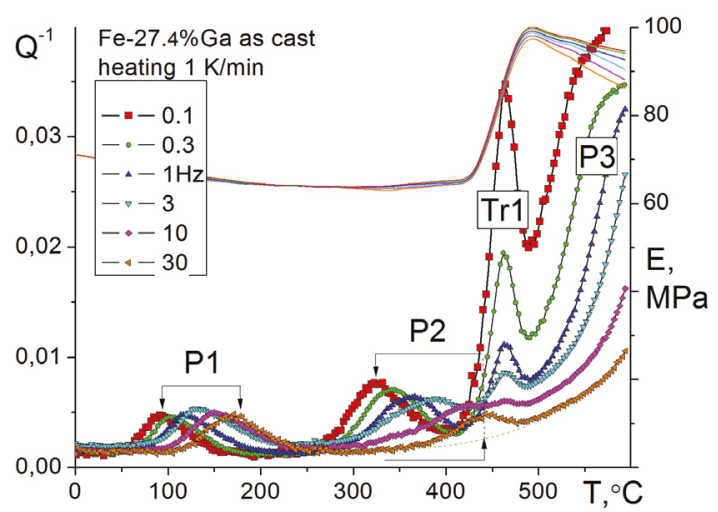

a)

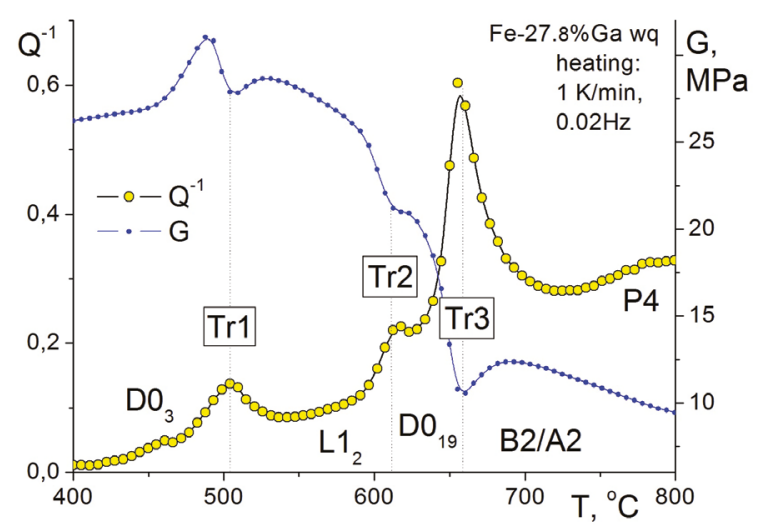

b)

Figure 1. TDIF curves measured at heating with $1 \mathrm{~K} / \mathrm{min}$ rate using bending (up to $600^{\circ} \mathrm{C}$, DMA Q800; left figure) and torsion (up to $800^{\circ} \mathrm{C}$, inverted pendulum; right figure) vibrations with different frequencies from 0.02 to $30 \mathrm{~Hz}$ (see in figures). 


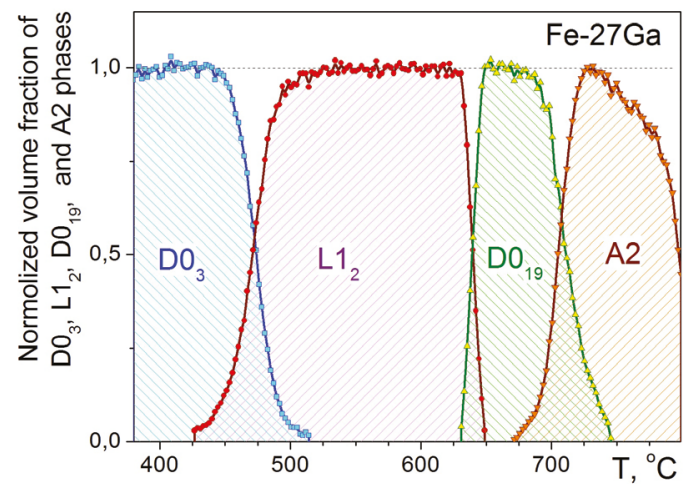

a)

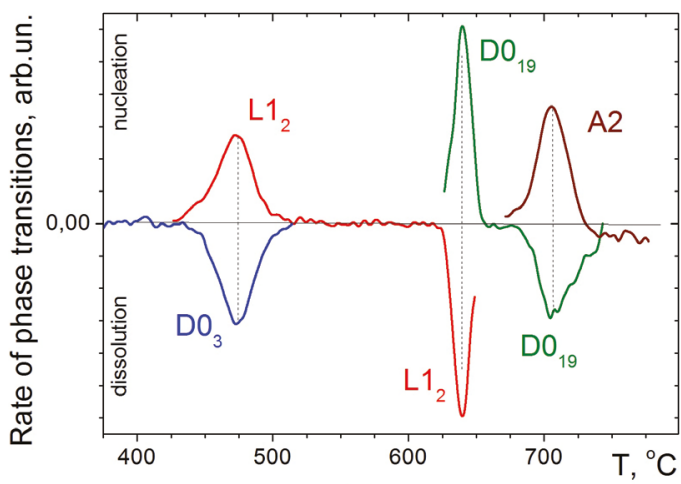

b)

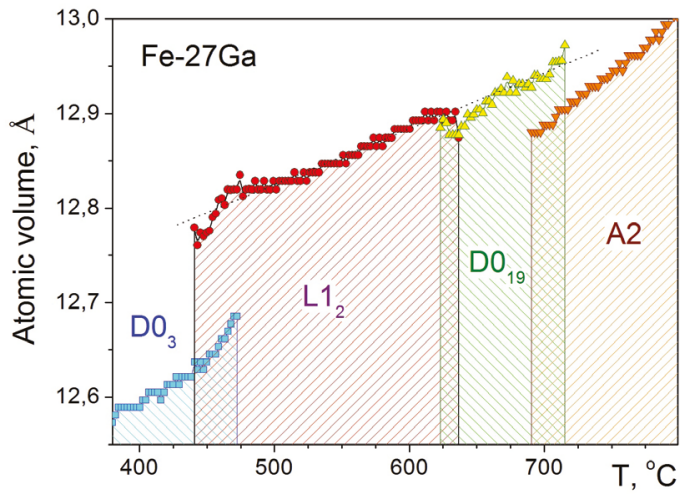

c)

Figure 2. Phase transitions in the Fe-27.0Ga (a) samples heated with a rate of $2 \mathrm{~K} / \mathrm{min}$ obtained by normalizing the intensities of the characteristic diffraction peaks at their maximum values (reduction of the $\mathrm{A} 2$ phase above $700^{\circ} \mathrm{C}$ is associated with a gradual decrease in the intensity of the magnetic contribution to the peaks); (b) First derivatives of the above curves-the rate of the phase transitions-illustrate the rate of growth and the dissolution of different phases. (c) Atomic volumes of the phases in the same temperature range.

\subsubsection{The $\mathrm{DO}_{3}$ to $\mathrm{LI}_{2}$ transition (Tr1) and tailoring magnetostriction of the alloy}

The relatively low-temperature $\mathrm{DO}_{3}$ to $\mathrm{L}_{2}$ transition ( $\operatorname{Tr} 1)$ was mainly studied using DMA equipment with varying frequencies from 0.1 to $30 \mathrm{~Hz}$. An example of these tests is given in Fig. 1a. The dependence of the Tr1-peak height on frequency and the heating rate is plotted in Fig. 3a. The dependence:

$$
\mathrm{Q}_{\mathrm{Tr}}^{-1}=\frac{\mathrm{k}}{\mathrm{J}} \frac{\mathrm{dn}}{\mathrm{dT}} \frac{\mathrm{T}}{\omega^{\mathrm{m}}},
$$

where $\omega=2 \pi f$ is the frequency, $(\mathrm{dn} / \mathrm{dT})$ is the volume fraction of the transformed phase during the transition with an increase in $\mathrm{T}$ - temperature, $\mathrm{J}$ is compliance, $\mathrm{k}$ and $\mathrm{m}$ are coefficients where $\mathrm{m} \approx 0.4^{5}$ is typical for a shear (martensitelike) transition. In contrast with ${ }^{18}$, no evidence of martensite or any other phases were detected by our in situ neutron diffraction tests. Moreover, careful study of the $\mathrm{DO}_{3}$ to $\mathrm{L}_{2}$ transition shows that this transition goes through diffusion controlled disordering $\mathrm{D}_{3}$ to $\mathrm{A} 2$ followed by $\mathrm{A} 2$ to $\mathrm{A} 1$ transition and $\mathrm{L}_{2}$ ordering of the $\mathrm{A} 1$ phase $\mathrm{e}^{2,7}$. Significant local stresses that appeared at $\mathrm{DO}_{3}$ to $\mathrm{L}_{2}$ transition are strongly anisotropic and the average lattice deformation depends on $\mathrm{D}_{3}$ and $\mathrm{L}_{2}$ phase fraction ${ }^{2}$ : an increase of the volume fraction of the $\mathrm{L}_{2}$ phase leads to an increase of the stress mainly in the $\mathrm{D}_{3}$ phase, whereas the stress in the $\mathrm{L}_{2}$ phase remains at the same level, which is relatively high from the beginning of the appearance of this phase.

If during the TDIF tests the heating is interrupted at the temperature of the Tr1-peak maximum, i.e., at about $470-475^{\circ} \mathrm{C}$ (Fig. 3b), the IF level decreases with holding time. A similar effect, which is slower, occurs in NiTi- or $\mathrm{CuAl}$-based alloys with a thermoelastic martensite transition ${ }^{19}$. The isothermal in situ neutron diffraction tests carried out at the same temperature demonstrate practically the same rate for the $\mathrm{D}_{3}$ to $\mathrm{L}_{2}$ transition rate (see inset in Fig. 3b). Thus, the Tr1-peak height is, indeed, proportional to the transition rate for the $\mathrm{DO}_{3}$ to $\mathrm{Ll}_{2}$ reaction, as predicted by Eq. (1), both at instant heating and isothermal annealing.

Isothermal annealing in the range of the $\mathrm{DO}_{3}$ to $\mathrm{L}_{2}$ transition leads to another important consequence. Isothermal heat treatment offers an excellent opportunity to vary the magnetostriction values in the alloys by interrupting the transition to obtain a different ratio between the phases. The metastable $\mathrm{DO}_{3}$ and the equilibrium $\mathrm{Ll}_{2}$ phase are both ferromagnetic phases, but with a different sign of magnetostriction. The difference in the ratio between these phases in the same sample, as a result of incomplete $\mathrm{D} 0_{3}$ to $\mathrm{L1}_{2}$ transition, may change magnetostriction of the sample from positive (in $\mathrm{DO}_{3}$ ) to negative (in $\mathrm{Ll}_{2}$ ) values. This effect was recently reported by Chinese researchers ${ }^{1,20}$ and by the present authors ${ }^{2,7}$.

The domain wall motion of the $\mathrm{D}_{3}$ phase with positive magnetostriction results in elongation up to the maximum value at lower magnetic fields. With an increase in the magnetic field, the $\mathrm{L}_{2}$ phase with stronger magneto-crystalline anisotropy reduces magnetostriction and contracts the sample (Fig. 4). Annealing at 400 and $435^{\circ} \mathrm{C}$ leads to different dispersion 


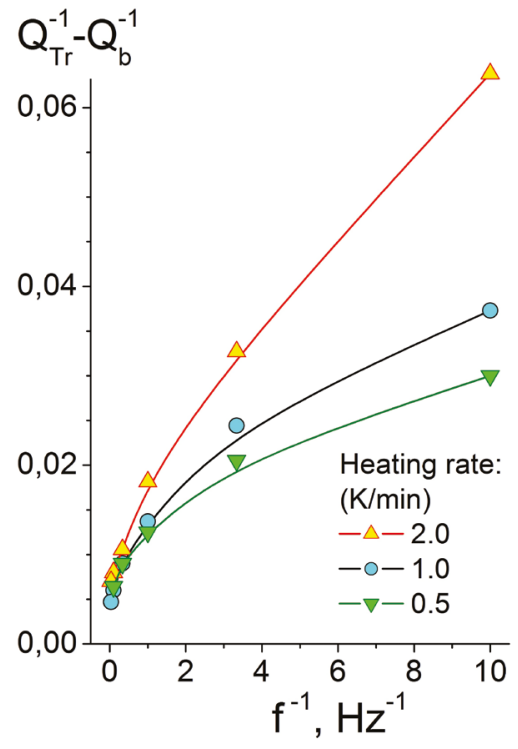

a)

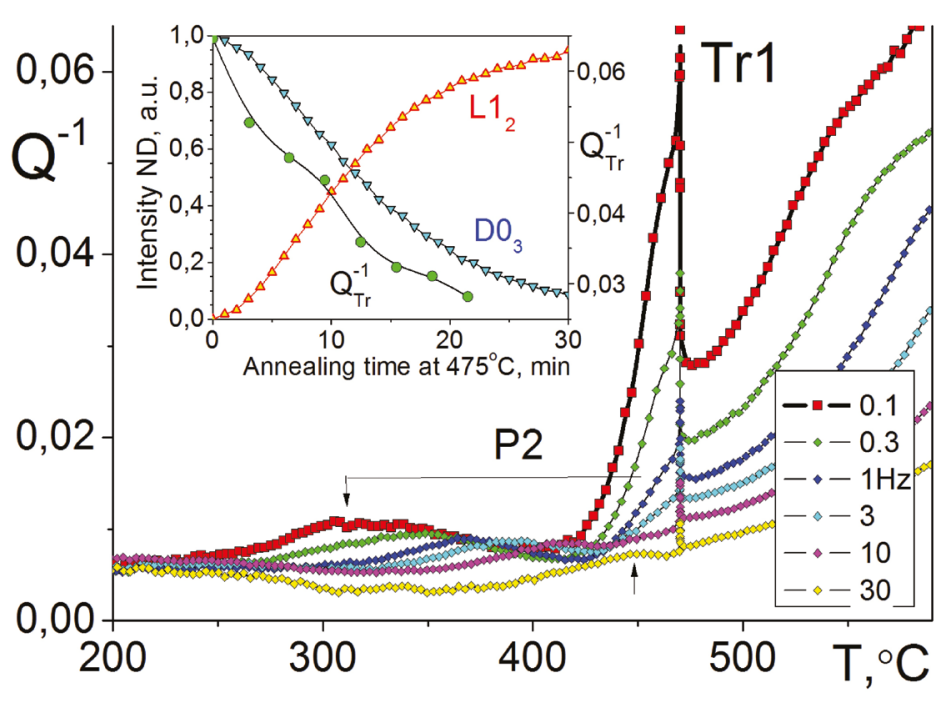

b)

Figure 3. a) The Tr1-peak height as a function of inverse frequency for three different heating rates; b) TDIF curve with interrupted heating at $470^{\circ} \mathrm{C}$. Inset: The decrease of the $\operatorname{Tr} 1$ peak as a function of time of isothermal annealing (green circles) and intensities of neutron diffraction lines from decaying $\mathrm{D} 0_{3}$ phase (blue down triangles) and growing $\mathrm{L}_{2}$ phase (red up triangles) under the same conditions.

of the $\mathrm{L}_{2}$ phase, which nuclei on the grain boundaries of the $\mathrm{D} 0_{3}$ phase. One can observe a different sensitivity of magnetostriction in the samples with differently dispersed $\mathrm{L}_{2}$ phase to the magnetic field. Both the maximum positive magnetostriction (red arrows) due to contribution of the $\mathrm{D}_{3}$ phase in low fields and the leveling-off magnetostriction values at higher magnetic field (black arrows) due to the compensating contribution of the $\mathrm{L} 1_{2}$ phase with negative magnetostriction occur at lower values of the applied field in the sample annealed at $400^{\circ} \mathrm{C}$ with a more dispersed $\mathrm{Ll}_{2}$ phase as compared with the samples annealed at $500^{\circ} \mathrm{C}$.

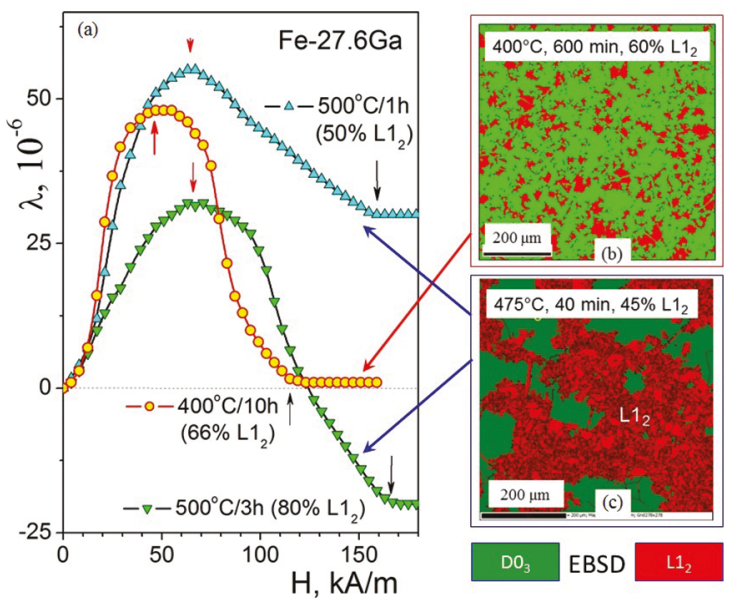

Figure 4. Magnetostriction vs applied field and different dispersion of the $\mathrm{L}_{2}$ (red color) phase at 400 (fine) and $475 / 500^{\circ} \mathrm{C}$ (coarse grains).
The magnetic domain structure in the Fe-27.8Ga sample annealed at $400{ }^{\circ} \mathrm{C}$ for $600 \mathrm{~min}$ was studied by MFM. The magnetic domain structure of the $\mathrm{D}_{3}$ and $\mathrm{L}_{2}$ phases is rather different: the $\mathrm{L}_{2}$ phase contains random and irregular magnetic domains, whereas the $\mathrm{D}_{3}$ phase has plate-like magnetic domains with a distinct magnetic substructure. Fig. $5 \mathrm{a}$ and $\mathrm{b}$ display the topography and the MFM images in a different region of the sample. Fig. 5c shows the enlarged region of the marked area (white rectangle), in Fig. 5b with irregular magnetic domain patterns in the $\mathrm{L1}_{2}$ phase and a large (width: 3-5 $\mu \mathrm{m}$, length: about $40 \mu \mathrm{m}$ ) well-aligned stripe domains are observed in the $\mathrm{D} 0_{3}$ phase. The domain walls in the well-aligned stripe domains are more easily movable as compared to the irregular magnetic domains.

\subsubsection{The $\mathrm{LI}_{2}$ to $\mathrm{DO}{ }_{19}$ transition}

According to the in situ neutron diffraction, the phase transition between two closed packed phases is not accompanied by a change in the atomic volume and a pronounced increase in internal stresses. Moreover, heating of the sample above the $\mathrm{D}_{3}$ to $\mathrm{L}_{2}$ transition leads to a smooth decrease in microstresses in the sample ${ }^{21}$. Thus, most probably, the IF peak ( $\mathrm{Tr} 2$ ) in the case of the $\mathrm{L}_{2} \rightarrow$ $\mathrm{D}_{19}$ (or fcc $\rightarrow$ hcp) phase transition is due to a long-range motion of the Shockley dislocation, which assists transition between these two closed packed phases. In accordance with our neutron diffraction data, the $\mathrm{L}_{2} \rightarrow \mathrm{D}_{19}$ transition also proceeds via disordering: $\mathrm{L1}_{2} \rightarrow \mathrm{A} 1 \rightarrow \mathrm{A} 3 \rightarrow \mathrm{D}_{19}$ (these data are not presented in this paper). 


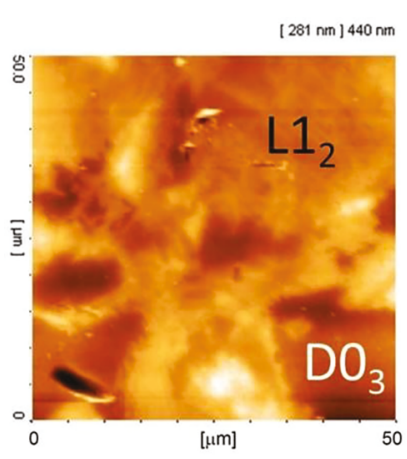

a

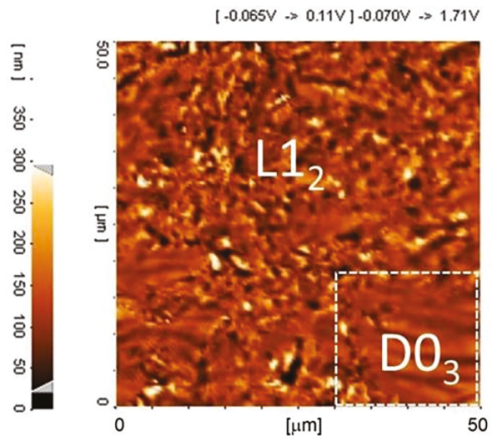

b

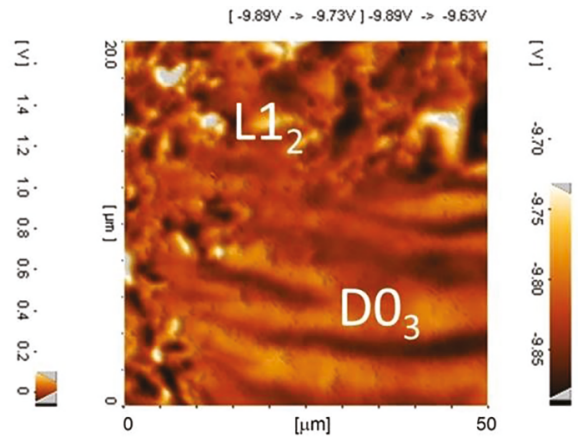

C

Figure 5. Topography (a), low magnification MFM (b), and high magnification MFM (c) images of the Fe-27.8Ga sample annealed at $400{ }^{\circ} \mathrm{C}$ for $600 \mathrm{~min}$ showing $\mathrm{DO}_{3}$ (darker contrast) and $\mathrm{L1}_{2}$ (brighter contrast) phases.

We carried out several tests with five frequencies (Fig. 6a) and four heating rates (Fig. 6b) in several subsequent runs, with the peak height changing roughly in agreement with Eq. (1). The effect of the frequency on the transient component of the martensitic transition peak for fcc - hcp transition in pure Co was studied by Bidaux et al. ${ }^{22}$. They demonstrated that the main (transient) component of internal friction must depend on $\dot{T} / \omega \sigma^{23}$. An increase in the heating rate expectably leads to a shift of these diffusion controlled phase transitions to a higher temperature: the red dash lines for $0.5 \mathrm{~K} / \mathrm{min}$ and the blue dot lines for $3 \mathrm{~K} / \mathrm{min}$ show the range of existence of $\mathrm{D} 0_{19}$ phase in Fig. $6 \mathrm{~b}$.

\subsubsection{The $D 0_{19}$ to A2 transition}

The effect of this first-order phase transition is similar to that of discussed in section 3.1.2. Here, we can only specify that it is the $\mathrm{D}_{19} \rightarrow \mathrm{B} 2 \rightarrow \mathrm{A} 2$ transition. According to the in situ neutron diffraction study, if the heating rate is $2 \mathrm{~K} /$ min, there is not enough time for the $\mathrm{B} 2 \rightarrow \mathrm{A} 2$ reaction, whereas in the case of heating with a rate of $1 \mathrm{~K} / \mathrm{min}$, the neutron diffraction detects weak $\mathrm{B} 2$ ordering of the A2 phase, as discussed $\mathrm{in}^{6}$. The $\operatorname{Tr} 3$ peak splitting with an increase in the heating rate (Fig. $6 \mathrm{~b}$ ) can be related to this effect, i.e., to the formation of the $\mathrm{A} 2$ phase from $\mathrm{D} 0_{19}$ followed by a $\mathrm{B} 2 \rightarrow \mathrm{A} 2$ reaction. This effect needs a more careful study in future. A little IF peak at about $800^{\circ} \mathrm{C}$, which probably corresponds to the $\mathrm{B} 2 \rightarrow \mathrm{A} 2$ reaction that is in agreement with the equilibrium phase diagram, is not included in Fig. 6.

It is important to note that each time the transient IF peak is associated with a modulus dip. The presence of a drop in the elastic shear modulus witnesses a strong coupling between the stress and the anelastic strain, which means that, similarly to the martensitic transformation, the phase interfaces propagate during the transformation. Misfit dislocations migration may also contribute. From the temperature of the modulus dip and considering the precision of the measurements, we cannot state that some precursor soft mode is activating the transformation.

\subsection{Doping $\mathrm{Fe}-27 \mathrm{Ga}$ by $\mathrm{Tb}$}

Doping the Fe-27Ga alloys by $\mathrm{Tb}$ significantly increases saturation magnetostriction in the as cast alloy with the $\mathrm{D}_{3}$ structure and slows down the $\mathrm{D}_{3}$ to $\mathrm{L}_{2}$ transition both upon isothermal annealing (Fig. 7a) and instant heating (Fig. $7 \mathrm{~b})$. The effect of $\mathrm{Tb}$ on magnetostriction is supposed to be caused by enhancing a tetragonal distortion in the nano-sized ordered domains in the bec derivative lattice. The effect of $\mathrm{Tb}$ on the kinetics of the phase transition from bcc to fcc derivative lattice in the $\mathrm{Fe}-\mathrm{Ga}$ alloys was also confirmed by neutron scattering ${ }^{13,24}$. Consequently, $\mathrm{Tb}$ smears the $\mathrm{D} 0_{3}$ to $\mathrm{L}_{2}$ transition, shifts it to higher temperatures and decreases a volume fraction of the $\mathrm{L}_{2}$ phase at constant heating ${ }^{13}$. As a result, the Tr1-peak is not well recorded in most of our tests of the Tb-doped Fe-27Ga alloys. In contrast, the Tr2- and Tr3-peaks at higher temperatures are well recorded (Fig. 7). Their temperature positions are in excellent agreement with the $\mathrm{L}_{2}$ to $\mathrm{D} 0_{19}$ and $\mathrm{D} 0_{19}$ to $\mathrm{B} 2$ transitions similarly to this effect in the binary alloys. In contrast with the binary alloys, in the Tb-doped samples, the $\mathrm{L}_{2}$ and $\mathrm{D} 0_{19}$ phases coexist with the $\mathrm{B} 2$ phase (the volume fraction of the $\mathrm{B} 2$ phase is at least twice larger as compared with these closed packed structures), as the $\mathrm{DO}_{3}$ to $\mathrm{L}_{2}$ transition is not complete in the alloys doped by $\mathrm{Tb}$.

The apparent activation parameters of the $\mathrm{P} 3$ peak are: $\mathrm{H}=2.5 \mathrm{eV}, \tau_{0}=2.5 \times 10^{-14}$, which is typical for a point defect peak with a broadening parameter $\approx 1$. Our estimation of the activation energy for the $\mathrm{P} 4$ peak gives a value of $2.84 \mathrm{eV}$, which is close to those for Fe-Al- $\mathrm{Si}\left(\mathrm{H}=2.7 \mathrm{eV}^{25}\right)$ and $\mathrm{Fe}-\mathrm{Al}$ $\mathrm{Cr}\left(\mathrm{H}=2.3-2.5 \mathrm{eV}^{26}, 2.7-2.9 \mathrm{eV}^{27}\right)$ alloys early reported i ${ }^{25-27}$ and assigned to dislocation peaks at elevated temperatures. The peak height in all these systems significantly increases when it is measured at cooling (not shown in the figure) as compared with the measurements at heating. In the ordered alloys, independently of the type of order, the mobility of the dislocations and the grain boundaries is significantly reduced, which results in low values of damping. In contrast, in the disordered state after heating to high temperatures and 


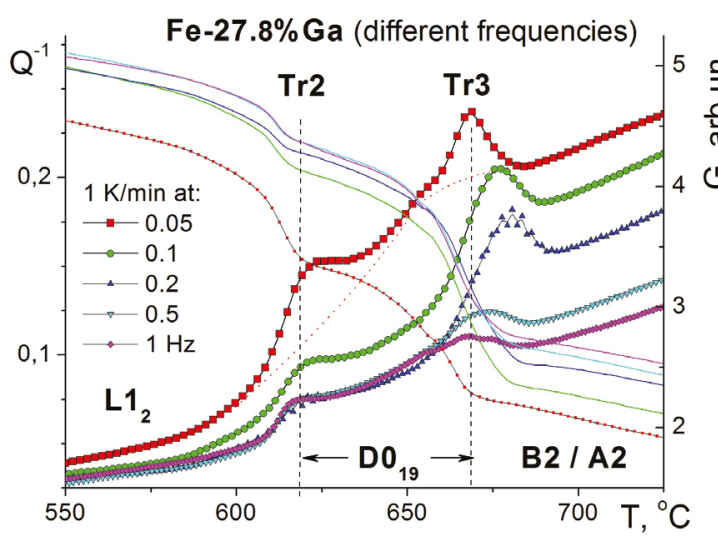

a)

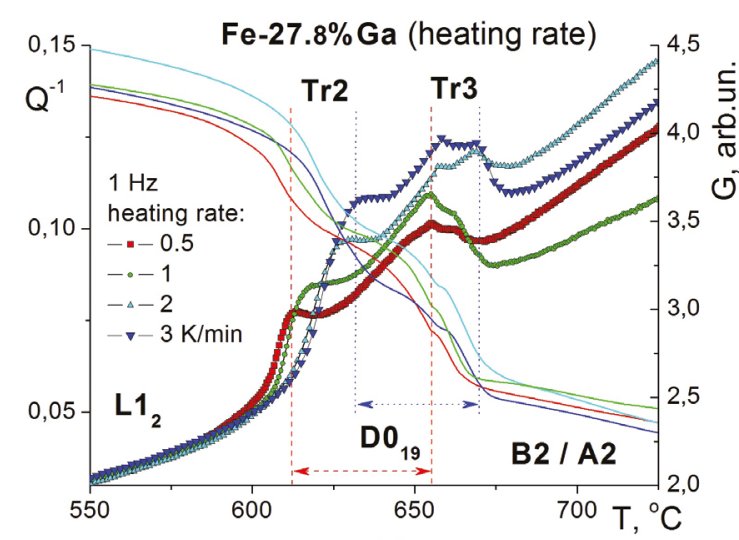

b)

Figure 6. Second and third transition peaks in the Fe-27.8Ga sample: a) influence of the frequency of vibrations (from 0.05 to $1 \mathrm{~Hz}$, heating rate $1 \mathrm{~K} / \mathrm{min}$ ), b) influence of the heating rate (from 0.5 to $3 \mathrm{~K} / \mathrm{min}, 1 \mathrm{~Hz}$ ).

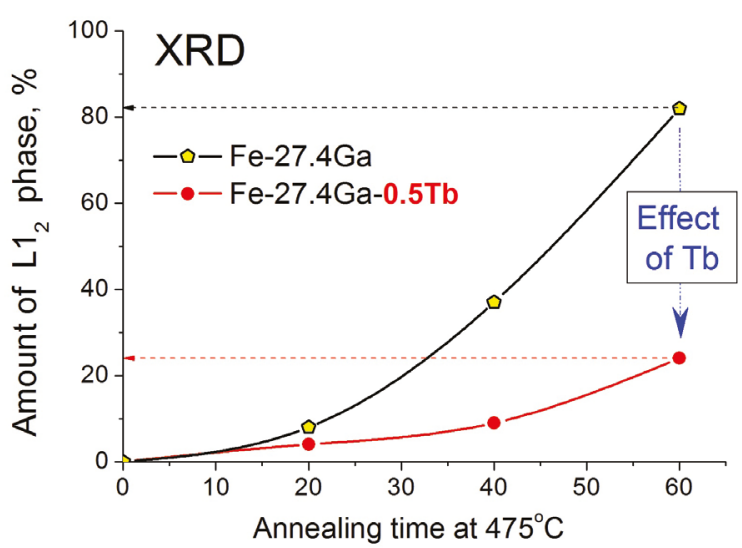

a)

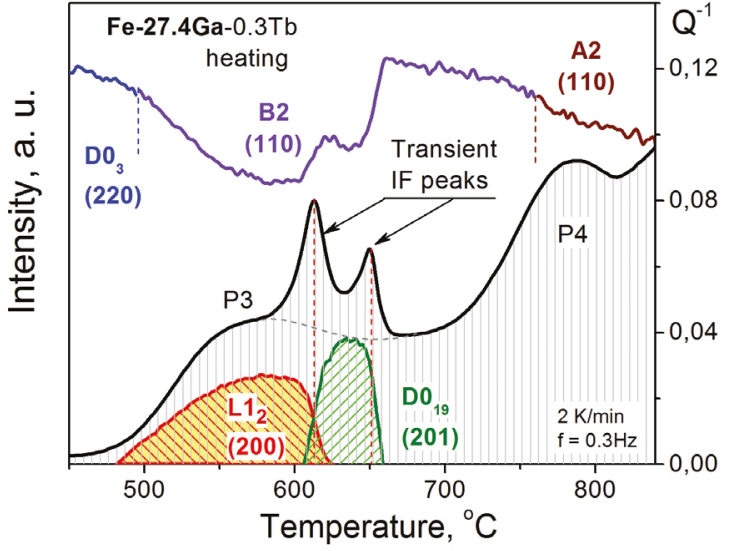

b)

Figure 7. (a) Amount of $\mathrm{L}_{2}$ phase determined by X-ray analysis after isothermal annealing in the Fe-27.4Ga (yellow circles) and in the Fe-27.4Ga-0.5Tb (red circles) samples; (b) Phase transitions in the Fe-27.4Ga-0.3Tb sample according to the in situ neutron diffraction (Intersity, left Y-axe) and TDIF (black curve corresponds to right Y-axe).

subsequent cooling, the dislocation and the grain boundary mobility increase, which leads to an increase in damping as compared with the ordered state ${ }^{28}$. Thus, we can preliminary attribute the $\mathrm{P} 4$ peak to dislocation effects.

\section{Conclusions}

Three transient IF effects or peaks are recorded in the studied Fe-Ga alloys. These peaks correspond to the $\mathrm{D}_{3} \rightarrow$ $\mathrm{L}_{2} \rightarrow \mathrm{D}_{19} \rightarrow \mathrm{A} 2$ (or B2) phase transitions. The transient peaks (the best studied is the $\operatorname{Tr} 1$ peak of the $\mathrm{D}_{3} \rightarrow \mathrm{Ll}_{2}$ transition in the binary alloys) have similar features with respect to transient peaks in the thermoelastic martensitic transition. Nevertheless, the in situ neutron diffraction does not support the idea about the appearance of the tetragonal phases by shear deformation. Moreover, the diffusion controlled disordering-ordering processes are confirmed. The $\mathrm{DO}_{3} \rightarrow \mathrm{Ll}_{2}$ and the $\mathrm{D}_{19} \rightarrow \mathrm{A} 2$ (or B2) phase transitions occur with a change in the atomic volume and a rise of the local stresses and strains, which lead to these anelastic peaks. The presence of the dips, i.e., shear modulus minima at the transition means that a softening of the elastic constants in some directions triggers the transition or maybe just a dislocation motion. One should note the pre-transition hardening that is better observed in the pendulum before the $\mathrm{DO}_{3} \rightarrow \mathrm{L}_{2}$ transition. This behaviour is probably due to the metastable nature of the $\mathrm{D}_{3}$ phase.

The $\mathrm{Ll}_{2} \rightarrow \mathrm{D} 0_{19}$ phase transitions are accompanied neither by a change in the atomic volume nor by a significant rise of the local strains. Most probably, the IF peak in this case is due to a long-range motion of the Shockley dislocation that assists this transition.

The $\mathrm{D}_{3} \rightarrow \mathrm{Ll}_{2}$ phase transition is a unique tool to vary magnetostriction in the Fe-27Ga type alloys. The parameters of magnetization vs. field curves depend on the dispersion of the $\mathrm{L}_{2}$ phase in the two-phase $\left(\mathrm{DO}_{3}+\mathrm{L}_{2}\right)$ samples. 


\section{Acknowledgements}

I.S.G. is grateful to NUST MISiS (5-100 Program) for financial support of his participation at ICIFMS-18 conference. Part of this study was carried out within RFBR project: 18-58-53032. The authors thank Mr. J. Cifre and Dr. V. Cheverikin for help with DMA tests and SEM EBSD work, Dr. E. Bazanova for her critical reading of the manuscript.

\section{References}

1. Gou J, Liu X, Wu K, Wang Y, Hu S, Zhao H, et al. Tailoring magnetostriction sign of ferromagnetic composite by increasing magnetic field strength. Applied Physics Letters. 2016;109(8):082404.

2. Palacheva VV, Emdadi A, Emeis F, Bobrikov IA, Balagurov $\mathrm{AM}$, Divinski SV, et al. Phase transitions as a tool for tailoring magnetostriction in intrinsic Fe-Ga composites. Acta Materialia. 2017;130:229-239.

3. Lograsso TA, Ross AR, Schlagel DL, Clark AE, Wun-Fogle M. Structural transformations in quenched Fe-Ga alloys. Journal of Alloys and Compounds. 2003;350(1-2):95-101.

4. Datta S, Huang M, Raim J, Lograsso TA, Flatau AB. Effect of thermal history and gallium content on magneto-mechanical properties of iron-gallium alloys. Materials Science and Engineering: A. 2006;435-436:221-227.

5. Golovin IS, Palacheva VV, Bazlov AI, Cifre J, Nollmann N, Divinski SV, et al. Diffusionless nature of $\mathrm{DO}_{3} \rightarrow \mathrm{L1}_{2}$ transition in $\mathrm{Fe}_{3} \mathrm{Ga}$ alloys. Journal of Alloys and Compounds. 2016;656:897902 .

6. Golovin IS, Balagurov AM, Palacheva VV, Bobrikov IA, Zlokazov VB. In-situ neutron diffraction study of bulk phase transitions in Fe-27Ga alloys. Materials \& Design. 2016;98:113-119.

7. Emdadi A, Palacheva VV, Cheverikin VV, Divinski S, Wilde $\mathrm{G}$, Golovin IS. Structure and magnetic properties of Fe-Ga alloys doped by Tb. Journal of Alloys and Compounds. 2018;758:214-223.

8. Golovin IS. Anelasticity of Fe-Ga based alloys. Materials \& Design. 2015;88:577-587.

9. Jiang L, Yang J, Hao H, Zhang G, Wu S, Chen Y, et al. Giant enhancement in the magnetostrictive effect of FeGa alloys doped with low levels of terbium. Applied Physics Letters. 2013;102(22):222409.

10. Meng C, Jiang C. Magnetostriction of a $\mathrm{Fe}_{83} \mathrm{Ga}_{17}$ single crystal slightly doped with Tb. Scripta Materialia. 2016;114:9-12.

11. Meng C, Wu Y, Jiang C. Design of high ductility FeGa magnetostrictive alloys: Tb doping and directional solidification. Materials \& Design. 2017;130:183-189.

12. Balagurov AM, Bobrikov IA, Golovin IS, Cheverikin VV, Golovin SA. Stabilization of bcc-born phases in Fe-27Ga by adding $\mathrm{Tb}$ : Comparative in situ neutron diffraction study. Materials Letters. 2016;181:67-70.

13. Golovin IS, Balagurov AM, Palacheva VV, Emdadi A, Bobrikov IA, Churyumov AY, et al. Influence of $\mathrm{Tb}$ on structure and properties of $\mathrm{Fe}-19 \% \mathrm{Ga}$ and $\mathrm{Fe}-27 \% \mathrm{Ga}$ alloys. Journal of Alloys and Compounds. 2017;707:51-56.

14. Emdadi A, Palacheva VV, Balagurov AM, Bobrikov IA, Cheverikin VV, Cifre J, et al. Tb-dependent phase transitions in Fe-Ga functional alloys. Intermetallics. 2018;93:55-62.

15. Li X, Bao X, Yu X, Gao X. Magnetostriction enhancement of $\mathrm{Fe}_{7} 3 \mathrm{Ga}_{27}$ alloy by magnetic field annealing. Scripta Materialia. 2018;147:64-68.

16. Balagurov AM. Scientific Reviews: High-resolution Fourier diffraction at the IBR-2 reactor. Neutron News. 2005;16(3):8-12.

17. Boyer SAE, Gerland M, Rivière A, Cifre J, Palacheva VV, Mikhaylovskaya AV, et al. Anelasticity of the Fe-Ga alloys in the range of Zener relaxation. Journal of Alloys and Compounds. 2018;730:424-433.

18. Lin YC, Lin CF. Effects of phase transformation on the microstructures and magnetostriction of $\mathrm{Fe}-\mathrm{Ga}$ and $\mathrm{Fe}-\mathrm{Ga}-\mathrm{Zn}$ ferromagnetic shape memory alloys. Journal of Applied Physics. 2015;117(17):17A920.

19. Schaller R, Fantozzi G, Gremaud G, eds. Mechanical Spectroscopy Q-1 2001 With Applications to Materials Science. Zurich: Trans Tech Publications; 2001.

20. Ma T, Gou J, Hu S, Liu X, Wu C, Ren S, et al. Highly thermal-stable ferromagnetism by a natural composite. Nature Communications. 2017;8:13937. DOI: 10.1038/ncomms13937

21. Balagurov AM, Golovin IS, Bobrikov IA, Palacheva VV, Sumnikov SV, Zlokazov VB. Comparative study of structural phase transitions in bulk and powdered $\mathrm{Fe}-27 \mathrm{Ga}$ alloy by real-time neutron thermodiffractometry. Journal of Applied Crystallography. 2017;50:198-210.

22. Bidaux J, Schaller R, Benoit W. Internal friction associated with the allotropic transformation of cobalt. Journal de Physique Colloques. 1985;46(C10):C10-601-C10-604.

23. Bidaux JE, Gremaud G, Benoit W. Transient Internal Friction and Martensitic Phase Transformations. Materials Science Forum. 1993;119-121:299-304.

24. Golovin IS, Emdadi A, Balagurov AM, Bobrikov IA, Cifre J, Zadorozhnyy MY, et al. Anelasticity of iron-aluminide $\mathrm{Fe}_{3} \mathrm{Al}$ type single and polycrystals. Journal of Alloys and Compounds. 2018;746:660-669.

25. Lambri OA, Pérez-Landazábal JI, Cuello GJ, Cano JA, Recarte $\mathrm{V}$, Siemers C, et al. Mechanical Spectroscopy in Fe-Al-Si alloys at elevated temperatures. Journal of Alloys and Compounds. 2009;468(1-2):96-102.

26. Lambri OA, Pérez-Landazábal JI, Recarte V, Cuello GJ, Golovin IS. Order controlled dislocations and grain boundary mobility in Fe-Al-Cr alloys. Journal of Alloys and Compounds. 2012;537:117-122.

27. Golovin IS, Rivière A. Anelasticity in Fe-Al-Cr alloys at elevated temperatures. Materials Science and Engineering. A. 2009;521-522:67-72.

28. Golovin IS, Balagurov AM. Structure Induced Anelasticity in Iron Intermetallic Compounds and Alloys. Materials Research Foundations. 2018;30:256 p. 